\title{
Nanoscale
}

Check for updates

Cite this: Nanoscale, 2021, 13, 19966

\section{Non-equilibrium diffusion of dark excitons in atomically thin semiconductors $\uparrow$}

\author{
Roberto Rosati, (iD *a Koloman Wagner, (iD b,f Samuel Brem, (iD) a \\ Raül Perea-Causín, (D) c Jonas D. Ziegler, (ID b,f Jonas Zipfel, (D) b,g \\ Takashi Taniguchi, (D) ${ }^{d}$ Kenji Watanabe, (D) e Alexey Chernikov (D) ${ }^{b, f}$ and \\ Ermin Malic (D) a,c
}

\begin{abstract}
Atomically thin semiconductors provide an excellent platform to study intriguing many-particle physics of tightly-bound excitons. In particular, the properties of tungsten-based transition metal dichalcogenides are determined by a complex manifold of bright and dark exciton states. While dark excitons are known to dominate the relaxation dynamics and low-temperature photoluminescence, their impact on the spatial propagation of excitons has remained elusive. In our joint theory-experiment study, we address this intriguing regime of dark state transport by resolving the spatio-temporal exciton dynamics in $\mathrm{hBN}$-encapsulated $\mathrm{WSe}_{2}$ monolayers after resonant excitation. We find clear evidence of an unconventional, time-dependent diffusion during the first tens of picoseconds, exhibiting strong deviation from the steady-state propagation. Dark exciton states are initially populated by phonon emission from the bright states, resulting in creation of hot (unequilibrated) excitons whose rapid expansion leads to a transient increase of the diffusion coefficient by more than one order of magnitude. These findings are relevant for both fundamental understanding of the spatio-temporal exciton dynamics in atomically thin materials as well as their technological application by enabling rapid diffusion.
\end{abstract}

Received 21st September 2021 Accepted 13th November 2021 DOI: $10.1039 / \mathrm{d} 1 \mathrm{nr} 06230 \mathrm{a}$

rsc.li/nanoscale light due to either spin- or momentum-conservation. However, they are sufficiently long-lived and can recombine either through out-of-plane polarized or phonon-assisted emission resulting in pronounced PL signatures located energetically below the optically bright exciton. ${ }^{21-28}$

Following optical excitation resonant with bright $\mathrm{X}_{0}$ peak, energetically favorable dark states are populated on a sub-picosecond timescale via emission of phonons from the energetically higher bright states (Fig. 1(a)). ${ }^{61}$ Due to the mismatch between exciton valley separation and phonon energy, this results in hot dark excitons exhibiting a considerable excess energy, as recently evidenced in spectrally and temporally resolved PL spectra. ${ }^{29}$ Overheated excitons in an otherwise cold lattice represent a particularly interesting scenario that should directly lead to a rapid expansion of excitons, $c f$. Fig. 1(a). These non-equilibrated excitons appear independently from the excitation density - in contrast to longer-lived non-linear effects previously observed at room temperature such as non-conventional diffusion ${ }^{30}$ and halo formation ${ }^{31,32}$ or also phonon wind and drag phenomena. ${ }^{33}$ Interestingly, while spatio-temporal dynamics of excitons in TMD mono- and few-layer materials has attracted broad attention, ${ }^{30-42}$ only little is known regarding transient propagation of dark excitons, before they reach the regime of conventional, time-independent diffusion. A direct demonstration of a transient dark exciton diffusion observing hot exciton populations on a picosecond time scale has yet remained unexplored in monolayer materials. 
(a) bright
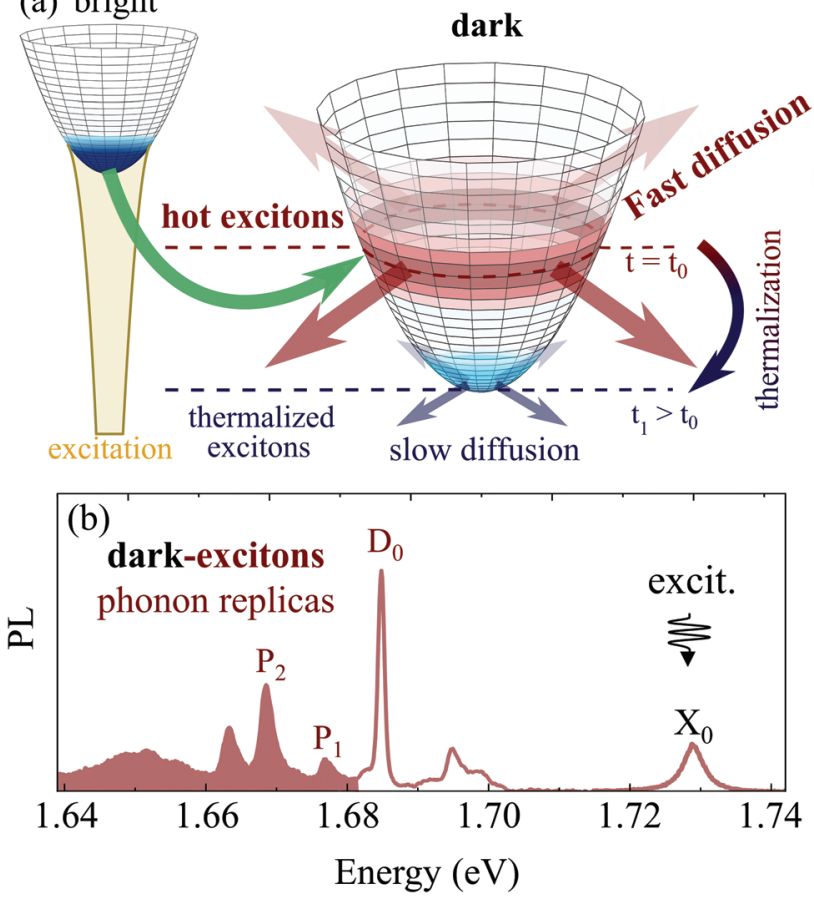

Fig. 1 Hot exciton formation. (a) Optically excited bright excitons scatter via emission of phonons into energetically lower momentumdark exciton states. The resulting excess energy of dark excitons leads to a fast, transient diffusion of the non-equilibrium population prior to thermal equilibrium. (b) Experimentally measured PL spectrum of hBNencapsulated $\mathrm{WSe}_{2}$ monolayer at $T=5 \mathrm{~K}$. Indicated are resonances stemming from the direct emission of bright excitons $\left(X_{0}\right)$ and out-ofplane polarized, spin-dark excitons $\left(D_{0}\right)$. Shaded area denotes the spectral range of phonon replicas, including those attributed to momentumdark states $\left(\mathrm{P}_{1}\right.$ and $\left.\mathrm{P}_{2}\right)$.

In this work, we address this intriguing exciton diffusion regime in a joint theory-experiment study, where we combine microscopic many-particle modeling with spatially and temporally resolved luminescence microscopy. While the diffusion after excitation in the continuum has been investigated in different materials, ${ }^{30,43-46}$ we focus here on optical excitation resonant to the deeply-bound bright exciton $\mathrm{x}_{0}, c f$. Fig. 1(b). We explicitly avoid high-excitation effects and focus on the linear regime. ${ }^{31-33,37}$ To suppress environmental disorder we employ hBN-encapsulated $\mathrm{WSe}_{2}$ monolayers, cooled down to cryogenic temperatures. These conditions allow us to take advantage of prolonged thermalization times ${ }^{29}$ but also gain direct optical access to dark excitons which recombine regardless of their center-of-mass momenta via characteristic, spectrally-narrow phonon sidebands, ${ }^{21-28} c f$. Fig. 1(b). This allows access to non-equilibrium regime of exciton diffusion via optical observation of dark excitons beyond commonly-studied bright states. In both theory and experiment we demonstrate an unconventional, time-dependent diffusion during thermalization and relaxation of dark excitons. We show a pronounced increase of the effective diffusion coefficient by more than one order of magnitude few picoseconds after the excitation, in contrast to the stationary case of locally-thermalized exciton population. The agreement between microscopic theory and spatio-temporal PL measurements provides a clear evidence of accelerated dark-exciton propagation in tungsten-based TMD monolayers.

\section{Microscopic modeling}

First, we present theoretical calculations with the goal to microscopically describe spatially and temporally resolved dynamics of the phonon-assisted emission from dark excitons. The energies and wavefunctions of bright and dark exciton states $^{7,13,16,17,22}$ are accessed via numerical solution of the Wannier equation ${ }^{8,47,48,62}$ including material-specific parameters for the electronic bandstructure. ${ }^{48}$ Due to the resonant optical excitation and considerable binding energies, we focus our study on the three energetically lowest 1s states, which in our calculations are $\mathrm{KK}^{\prime}$ excitons followed by $\mathrm{K} \Lambda$ and bright $\mathrm{KK}$ ones. Here, $\mathrm{K}, \mathrm{K}^{\prime}, \Lambda$ denote the high-symmetry points in the reciprocal space that host electrons and holes forming an exciton. We restrict our analysis to spin-allowed states emitting through low-energy phonon replicas, even as the emission from spin-forbidden states is also observed in experiments ${ }^{25,26,49,50}$ (e.g. $\mathrm{D}_{0}$ resonance in Fig. $1(\mathrm{~b})$ ). While spin-dark states are found to exhibit similar diffusion coefficients at longer timescales after relaxation, ${ }^{35,51}$ their larger formation times compete with the non-equilibrium processes studied here, in particular with a minor presence of phonon sidebands from spin-dark states in the first few tens of picoseconds. ${ }^{29}$

To obtain a microscopic access to the spatio-temporal exciton dynamics, we introduce the excitonic Wigner function $N_{\mathbf{Q}}^{\mathrm{v}}(\mathbf{r}, t)$, which provides the quasi-probability of finding excitons with momentum $\mathbf{Q}$ at time $t$ in position $\mathbf{r}$ and exciton valley $\mathrm{v}^{39}$ We derive its equation of motion using the Heisenberg equation and transforming into the Wigner representation. ${ }^{52}$ In the low-density regime it reads ${ }^{39}$

$$
\begin{aligned}
\dot{N}_{\mathbf{Q}}^{\mathrm{v}}(\mathbf{r}, t)= & \left(\frac{-\hbar \mathbf{Q}}{M_{\mathrm{v}}} \cdot \nabla-\gamma \delta_{\mathbf{Q}, 0} \delta_{\mathrm{v}, \mathrm{KK}}\right) N_{\mathbf{Q}}^{\mathrm{v}}(\mathbf{r}, t) \\
& +\Gamma_{\mathbf{Q} ; 0}^{\mathrm{v} ; \mathrm{KK}}\left|P_{0}(\mathbf{r}, t)\right|^{2}+\left.\dot{N}_{\mathbf{Q}}^{\mathrm{v}}(\mathbf{r}, t)\right|_{\mathrm{sc}} .
\end{aligned}
$$

The first term describes free propagation of excitons depending on the total mass $M_{\mathrm{v}}$ and the spatial gradient in the exciton occupation, resulting in faster spatial variations for more confined exciton distributions ( $c f$. ESI $\dagger$ ). The second term takes into account losses due to the radiative recombination $\gamma$ within the light cone $\left(\delta_{\mathbf{Q}, 0} \delta_{\mathrm{v}, \mathrm{KK}}\right)$. The third and fourth contributions represent phonon-assisted formation and dynamics of excitons, respectively.

For our study, we consider a short and confined optical pulse in resonance with bright exciton states: this initially $(t=$ 0 ) creates an excitonic polarization $P_{\mathbf{Q} \approx 0}(\mathbf{r}, t)$ in the light cone (i.e. "coherent excitons" 53,54 ), which can then be converted into population of "incoherent excitons" via scattering. In particular exciton-phonon scattering with the rates $\Gamma_{\mathbf{Q Q}^{\prime}}^{\mathrm{vv}^{\prime}}$ drives the formation of incoherent excitons also in other valleys via inter- 
valley mechanisms. ${ }^{5,56}$ Finally, the exciton-phonon interaction leading e.g. to thermalization is described via the Boltzmann scattering term $\left.\dot{N}_{\mathbf{Q}}^{\mathrm{v}}(\mathbf{r}, t)\right|_{\text {th }}=\Gamma_{\mathbf{Q}}^{\text {in,v }}(\mathbf{r}, t)-\Gamma_{\mathbf{Q}}^{\text {out,v }} N_{\mathbf{Q}}(\mathbf{r}, t)$ with phonon-driven in- and out-scattering rates $\Gamma_{\mathbf{Q}}^{\text {in/out,v }}(\mathbf{r}, t){ }^{57}$ Here, we neither consider non-thermal phonons nor Augerlike recombination processes, which can be described via additional non-linear terms in eqn (1), ${ }^{32}$ that become important only for strong excitations and can lead to phenomena, such as halo formation on comparatively long timescales. ${ }^{31-33}$ Thus, exciton-phonon scattering in the linear density regime provides the leading contribution to the interplay between energy relaxation and spatio-temporal dynamics, determining transient diffusion studied in this work.

The first contribution in eqn (1) alone leads to a ballistic exciton propagation, where each state moves in space along the direction of $\mathbf{Q}$ with a velocity proportional to $|\mathbf{Q}|$. Excitonphonon scattering (second line in eqn (1)), however, redistributes exciton occupation primarily toward states with smaller energies (energy-relaxation) and those of different orientation in the reciprocal space (momentum-relaxation). In contrast to the ballistic regime, these scattering processes lead to the typically slower, conventional exciton diffusion.

Different propagation regimes can be quantified by studying the evolution of the spatial width $w(t)$ of a given exciton distribution $N(\mathbf{r}, t)$. This is evaluated via $w^{2}(t)=\int \mathbf{r}^{2} N(\mathbf{r}, t) \mathrm{d} \mathbf{r} / \int N(\mathbf{r}, t) \mathrm{d} \mathbf{r}$, with the area $w^{2}$ corresponding for Gaussian distributions to the denominator in the exponent $\left(N(\mathbf{r}, t) \propto \exp \left[-x^{2} / w^{2}(t)\right]\right)$. In the ballistic regime, $w^{2}$ increases quadratically in time, while in the conventional diffusive regime the dependence is strictly linear. ${ }^{58}$ Deviations from this conventional linear law can be described by defining an effective time-dependent diffusion coefficient $D(t)=\frac{1}{4} \partial_{t} w^{2} \cdot{ }^{39}$ This coefficient typically converges to a constant $D=k_{\mathrm{B}} T \tau_{\mathrm{s}} / M_{\mathrm{X}}$, when the conventional diffusion is reached, with $\tau_{\mathrm{s}}$ being a state-independent scattering time. As we demonstrate in the following, the regime of unconventional, time-dependent diffusion can last for tens of picoseconds in $\mathrm{WSe}_{2}$ monolayers at cryogenic temperatures, where equilibration mechanisms are sufficiently slow.

\section{Results}

\subsection{Spatio-temporal exciton dynamics}

To investigate the spatially and temporally dependent optical response of hBN-encapsulated $\mathrm{WSe}_{2}$ monolayers, we solve the equation of motion for the Wigner function [eqn (1)] and the generalized Elliot formula for the $\mathrm{PL}(c f$. ESI $\dagger)$. In the calculations, we set the lattice temperature to $20 \mathrm{~K}$ and consider pulsed excitations confined to an initial spatial width of $w \approx$ $0.5 \mu \mathrm{m}$. In Fig. 2 we present an overview of the resulting energy-resolved spatial profiles of excitonic distributions (Fig. 2(a-c)) and phonon sidebands (Fig. 2(d-f)) at given times. This gives access to the relaxation of excitons in time and energy and associated evolution of PL spectra, while the result-
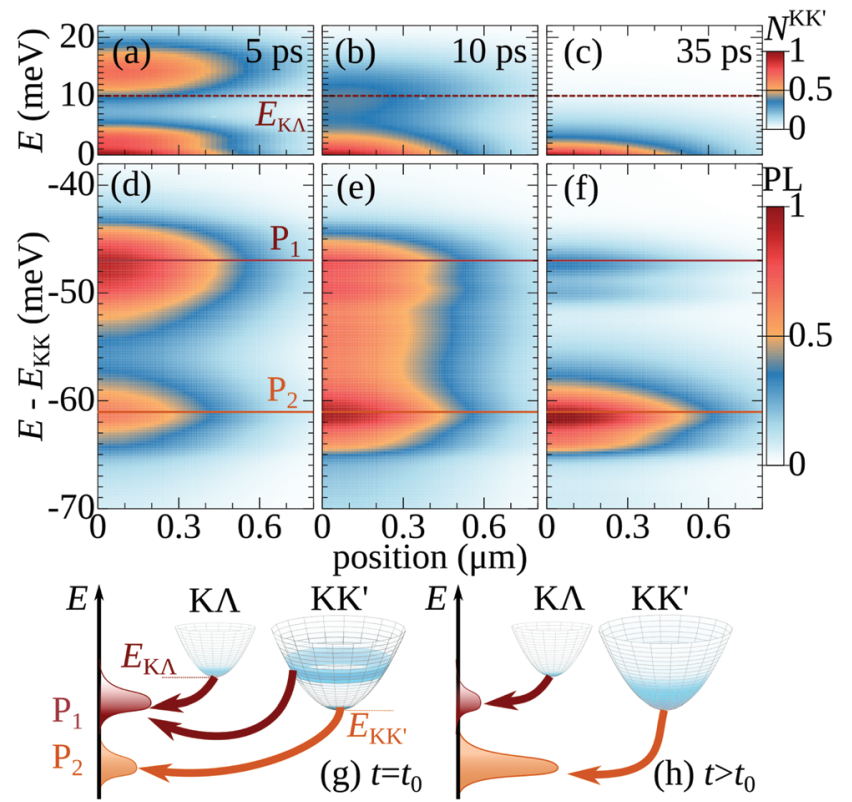

Fig. 2 Non-equilibrium excitons dynamics. (a)-(c) Spatio-temporal dynamics of momentum-dark KK' excitons at $20 \mathrm{~K}$ illustrating the interplay between exciton propagation away from the excitation spot and their thermalization towards thermal equilibrium. (d)-(f) Corresponding spatio-temporal evolution of the PL from momentum-dark excitons emitting through phonon-assisted recombination. The two phonon sidebands $P_{1}$ and $P_{2}$ can be traced back mainly to KK' excitons with some contribution from $\mathrm{K} \Lambda$ states at early times. Schematic illustration of the origin of phonon sidebands ( $\mathrm{g}$ ) directly after optical excitation and (h) after thermalization into an equilibrium distribution.

ing spatial propagation behavior is analyzed and discussed in detail in Fig. 3 and 4. Fig. 2(a-c) illustrate the resulting occupation of the energetically lowest momentum-dark $\mathrm{KK}^{\prime}$ excitons, $N_{\mathbf{Q}}^{\mathrm{KK}^{\prime}}(\mathbf{r}, t)$ at different times after the optical excitation. We focus on only one spatial direction due to rotational symmetry of the system.

Interestingly, the distributions of both excitons and PL in energy are notably different between the three considered times of 5, 10, and 35 ps after the excitation. In particular, after 5 ps (Fig. 2(a)) excitons are still far from the thermal equilibrium. We find a pronounced hot-exciton region, where excitons carry a considerable excess energy on the order of $15 \mathrm{meV}$. This energy is acquired after phonon-assisted relaxation from the optically-excited bright to the dark state, (Fig. 1) and stems from the difference between the bright-dark energy separation and the energy of the inter-valley phonons involved. These overheated excitons subsequently thermalize and cool down, losing their kinetic energy mainly via scattering with intravalley acoustic modes, so that the distribution starts spreading approximately $10 \mathrm{ps}$ after the excitation. After 20 to $30 \mathrm{ps}$, excitons finally form a Boltzmann distribution with a temperature corresponding to that of the lattice (Fig. 2(c)). The presented relaxation dynamics in momentum space is consistent with the case of a spatially-homogeneous excitation. ${ }^{29}$ For spatially-localized excitation, however, it has major implications for the exciton propagation, as discussed below. 

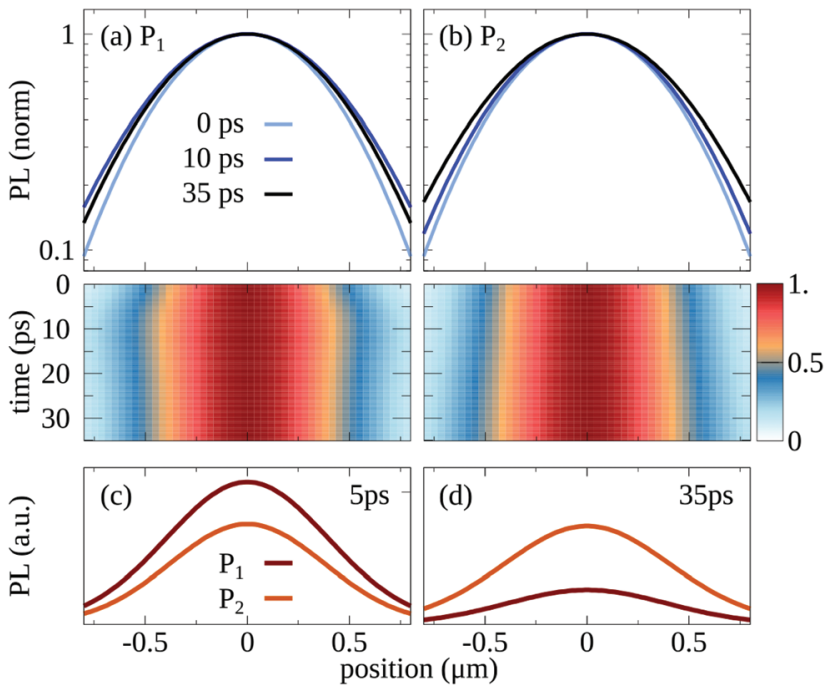

Fig. 3 Spatio-temporal dynamics of phonon sidebands. Exciton emission profiles at representative times (upper panel) and spatially and temporally resolved surface plots (lower panel) evaluated at the spectral position of the (a) $P_{1}$ and (b) $P_{2}$ sideband. It demonstrates both an initially accelerated (a) and slower (b) exciton propagation due to nonequilibrium $\mathrm{KK}^{\prime}$ excitons emitting predominantly at $\mathrm{P}_{1}$ first and subsequently at $P_{2}$ spectral positions, following relaxation. (c) and (d) Direct comparison between the spatial intensity of $P_{1}$ and $P_{2}$ phonon sidebands at different times (normalized w.r.t. $P_{2}$ ).

To obtain a key observable accessible in experiments, we present the corresponding spatially and spectrally dependent PL in Fig. 2(d-f). In the studied regime, it is dominated by phonon sidebands of momentum-dark excitons, located approximately 50 to $60 \mathrm{meV}$ below the energy of the bright $\mathrm{KK}$ exciton. $^{28}$ The majority of the PL signal is traced back to the $\mathrm{KK}^{\prime}$ excitons that recombine under emission of the zone-edge acoustic phonons with an energy around $15 \mathrm{meV} \cdot{ }^{5,56}$ Notably, phonon-assisted processes allow for all exciton states to emit, regardless of their center-of-mass momenta. As a consequence, the profiles in Fig. 2(d-f) largely follow the exciton distribution presented in Fig. $2(\mathrm{a}-\mathrm{c})$. In addition to $\mathrm{KK}^{\prime}$ sidebands, we also find contributions from $\mathrm{K} \Lambda$ excitons at early times (Fig. 2(g) and (h)) that partially overlap the emission from hot $\mathrm{KK}^{\prime}$ excitons with an excess energy of about $15 \mathrm{meV}$ (Fig. 2(a and d)). This overlap results in an initially more pronounced $\mathrm{P}_{1}$ sideband, $c f$. Fig. 2(d).

\subsection{Transient exciton diffusion}

In the following, we consider the consequences of hot dark excitons with high excess energies for the exciton diffusion. Spatio-temporal PL signals, evaluated at the energies of the two pronounced $\mathrm{P}_{1}$ and $\mathrm{P}_{2}$ phonon sidebands are presented in Fig. 3(a) and (b). We find a fast spatial broadening of the $\mathrm{P}_{1}$ signal in the first $10 \mathrm{ps}$ (reaching a transient diffusion coefficient $D$ of many 10's of $\mathrm{cm}^{2} \mathrm{~s}^{-1}, c f$. ESI $\dagger$ ). The driving force for the initial increased spatial broadening are hot KK' excitons, which emit light approximately at the same energy as the $\mathrm{K} \Lambda$ states (Fig. 2(g)). This initial spatial broadening of KK' excitons is much faster than that of $\mathrm{K} \Lambda$ excitons due to their higher excess energy, $c f$. Fig. 1(a), as further confirmed by calculating energy- and exciton-valley-specific diffusion coefficients $D_{\mathrm{E}, \mathrm{v}}$ ( $c f$. ESI $\dagger$ ). Over time, the spatial broadening slows down, and eventually results in a small narrowing, i.e. effectively negative diffusion: At $35 \mathrm{ps}$ the profile is narrower than at $10 \mathrm{ps}$, since former hot KK' excitons have cooled down. Hence, their signal does not contribute anymore to the emission in the spectral range of $\mathrm{P}_{1}, c f$. Fig. $2(\mathrm{~h})$ and ESI. $\dagger$

The situation in $\mathrm{P}_{2}$ is qualitatively different, exhibiting a much slower spatial broadening in the first 10 ps (Fig. 3(b)). Here, the main contribution of the PL signal stems from nearly thermalized $\mathrm{KK}^{\prime}$ excitons with a vanishing excess energy (Fig. 2(g)). As a consequence, the initial spatial broadening is considerably slower compared to $\mathrm{P}_{1}$. However, it lasts longer due to the cooling of hot $\mathrm{KK}^{\prime}$ excitons, which initially diffuse faster but emit spectrally away from $\mathrm{P}_{2}$. When they relax, their emission increasingly occurs in the spectral range of $\mathrm{P}_{2}$ that becomes spatially broader over time ( $c f$. ESI $\dagger$ ). This leads to a broader $\mathrm{P}_{2}$ profile compared to that of $\mathrm{P}_{1}$ at $35 \mathrm{ps}$. Finally, after a few tens of ps the stationary situation of conventional diffusion is recovered. Besides the qualitatively different diffusion behavior, $\mathrm{P}_{1}$ and $\mathrm{P}_{2}$ phonon sidebands also differ in their intensity with the signal in $\mathrm{P}_{1}$ being initially stronger (Fig. 3(c)) then weaker than $\mathrm{P}_{2}$ after approx. 10 ps (Fig. 3(d)). This reflects the cooling of hot $\mathrm{KK}^{\prime}$ excitons, whose PL consequently spectrally shift from $\mathrm{P}_{1}$ to $\mathrm{P}_{2}$ (Fig. 2 ( $\mathrm{g}$ and $\mathrm{h}$ )).

\subsection{Measurement of transient exciton diffusion}

To study the theoretically predicted impact of hot dark excitons on the transient diffusion, we perform measurements of spatially and temporally resolved $\mathrm{PL}$ on hBN-encapsulated $\mathrm{WSe}_{2}$ monolayers, cooled to liquid helium temperature. The samples are obtained by mechanical exfoliation and stamping of bulk crystals onto $\mathrm{SiO}_{2} / \mathrm{Si}$ substrates ${ }^{60}$ and allow for an effective suppression of the environmental disorder, thus offering clean access to the phonon-assisted emission from dark states. For excitation, we use a $80 \mathrm{MHz}, 140$ fs-pulsed Ti: sapphire source tuned into resonance conditions with the bright exciton $\mathrm{X}_{0}$ at $1.726 \mathrm{eV}$ and focused to a spot with a submicron diameter. The resulting emission is collected from a lateral cross-section and guided through an imaging spectrometer equipped with a mirror and a grating to provide spatial and spectral resolutions, respectively. For time-resolved detection we use a streak camera operated in the single-photoncounting mode. ${ }^{31}$

Spatially-resolved PL is acquired in the spectral region of the phonon sidebands including $\mathrm{P}_{1}$ and $\mathrm{P}_{2}$, i.e. approximately $50 \mathrm{meV}$ below the bright exciton resonance, $c f$. Fig. 1(b). A direct comparison between theoretically predicted and experimentally measured spatio-temporal PL is presented in Fig. 4. Representative PL profiles at 0,10 , and 35 ps after the excitation are presented in the upper panel of Fig. 4(a) for calculated and measured distributions. The lower panel shows corresponding spatially and temporally resolved surface plots of the emission. In addition, we present differential PL profiles 

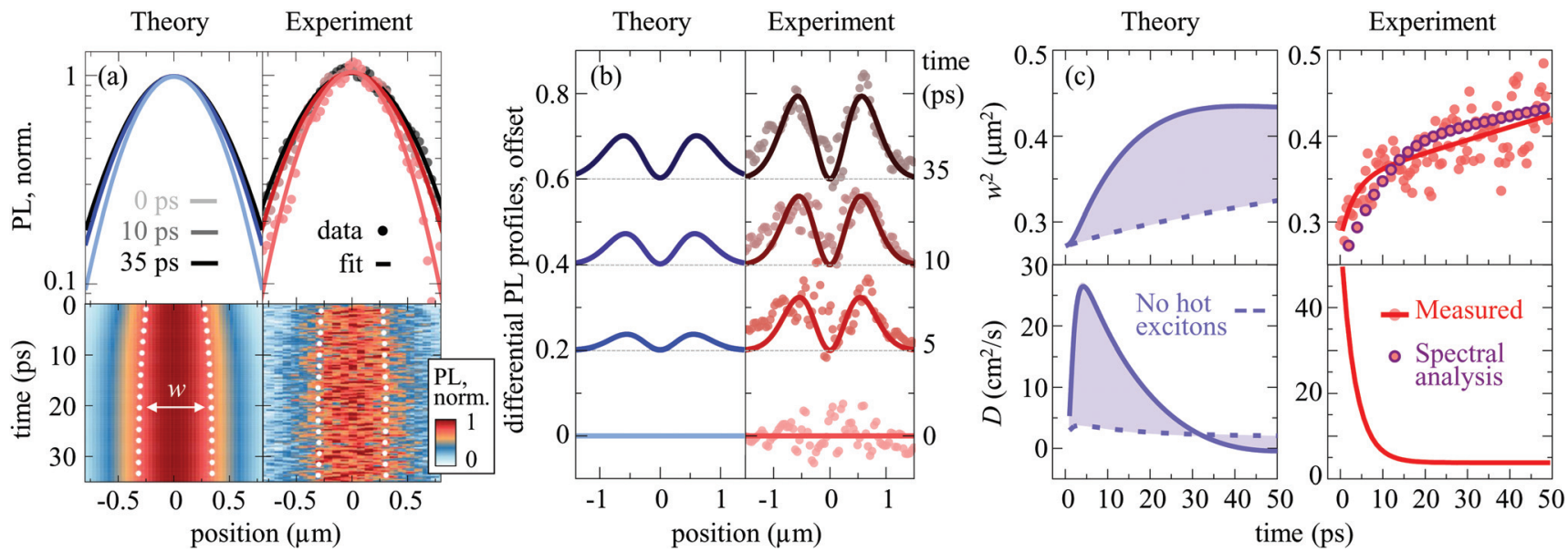

Fig. 4 Theory-experiment comparison of hot exciton diffusion. Calculated and measured representative spatial profiles of the PL at fixed times (upper panel) and two-dimensional spatially and temporally resolved PL (lower panel). The data is normalized to the maximum signal intensity at each time step. The solid lines in the upper experimental panel show Gaussian fits, while in the lower panel the white dashed lines show the extracted profile width $w(t)$. (b) Selected differential PL profiles at fixed times after the excitation $I_{\mathrm{PL}}(x, t)-I_{\mathrm{PL}}(x, t=0)$ obtained by subtracting the normalized, fitted lineshape of the initial profile at $t=0$ from the measured, normalized profiles at later times. The data is averaged over $0.08 \mu \mathrm{m}$ on the spatial axis and integrated over 4 ps in time. Differential fits by Gaussian peak functions are shown by solid lines. (c) Temporal evolution of the extracted squared spatial width $w^{2}$ and associated transient diffusion coefficient $D$ (upper and lower panel, respectively). In experiments, each data point corresponds to the automatically fitted width $w$ of measured PL profiles extracted from (a). In the calculations, the shaded area between full evolution (solid thin line) and assuming initial thermal equilibrium (dashed line) illustrates the impact of non-equilibrated, hot excitons.

in Fig. 4(b), corresponding to the relative change of the emission as compared to the initial one at $t=0$. Both theory and experiment show an initially fast spatial broadening in the first 10 ps followed by smaller variations. Finally, in Fig. 4(c) we show the extracted, time-dependent variance $w(t)^{2}$, obtained by automated procedures. The width $w(t)$ is further indicated in Fig. 4(a) by dashed white lines. Timedependent effective diffusion coefficient is then extracted from $D(t)=\frac{1}{4} \partial_{t} w(t)^{2}$ and presented in Fig. $4(\mathrm{c})$.

During the first 10's of ps we observe deviations from the standard diffusion law $w(t)^{2} \propto t$. This initially fast increase stems from rapidly diffusing hot excitons with high excess energies that later converges towards steady-state diffusion after exciton thermalization and cooling. The corresponding effective diffusion coefficients are found experimentally to be in the range of 30 to $50 \mathrm{~cm}^{2} \mathrm{~s}^{-1}$ immediately after optical excitation, more than an order of magnitude higher than the equilibrated value of about $3 \mathrm{~cm}^{2} \mathrm{~s}^{-1}$. The experimental findings agree overall very well with theoretical predictions. Theoretically predicted timescales are slightly longer and could be related to a slight underestimation of exciton-phonon matrix elements, eventual residual strain and weak unintentional free carrier concentrations in the studied materials. We note that the initial increase of the diffusion coefficient predicted by theory and reflecting an initial quasi-ballistic propagation ${ }^{39,59}$ could not be observed in the experiment due to the limited time-resolution. The initially rapid diffusion from hot excitons and a substantially slower propagation in the steady-state, inherently limited by the exciton scattering with linear acoustic phonons, are captured both in experiment and theory.
In addition, it is further instructive to consider a direct comparison of spectrally and spatially resolved data. In particular, we measure the spectral shifts $\Delta E(t)$ of the phonon sideband emission from the time-dependent PL spectrum in the region of $\mathrm{P}_{1}$ and $\mathrm{P}_{2}$ resonances ( $c f$. ESI $\dagger$ ). This can be interpreted as an excess energy affecting the diffusion coefficient via $D(t)=\left(\Delta E(t)+k_{\mathrm{B}} T\right) \times \tau / M_{\mathrm{X}}$, setting $T=5 \mathrm{~K}$ to the lattice temperature, the scattering time $\tau=2.8 \mathrm{ps}$ from the steadystate linewidth and $M_{\mathrm{X}}=0.75 m_{0} \cdot{ }^{48}$ As shown by the circles in Fig. 4(c), this results in a good agreement with the direct measurement of the time-dependent diffusion, further supporting the interpretation of hot exciton propagation.

The diffusion behavior is found to be drastically different when neglecting the impact of hot excitons, i.e. considering an initial exciton distribution at thermal equilibrium in our calculations, $c f$. the dashed lines in Fig. 4(c). Similarly to the case of bright $\mathrm{MoSe}_{2}$ monolayers (see ESI $\dagger$ ), without hot excitons there is no fast transient diffusion: we find a transient diffusion coefficient $D(t)<4 \mathrm{~cm}^{2} \mathrm{~s}^{-1}$, i.e. one order of magnitude smaller compared to the case with hot excitons. The timescale for the small maximum of $D(t)$ corresponds to $\tau$ and indicates the momentum relaxation after around one exciton-phonon scattering event. In contrast the energy relaxation in the real scenario requires emission of various (quasi-elastic) phonons: this results in the $10 \mathrm{ps}$ timescale with an intermediate regime between ballistic and diffusive exciton propagation. Furthermore, at approximately 40 ps after the optical excitation, we also predict small negative diffusion values (Fig. 4(c)) induced by the interstate thermalization and backscattering processes. ${ }^{39}$ This weak feature could not be resolved 
in the experiment at the current stage, motivating future studies. At later times approaching 100 ps, the regime of conventional diffusion is reached, so that dashed and solid lines converge to the same value of almost $2 \mathrm{~cm}^{2} \mathrm{~s}^{-1} \cdot{ }^{51}$

\section{Conclusions}

In summary, we have demonstrated non-equilibrium propagation of hot dark excitons in atomically thin semiconductors by combining microscopic many-particle theory with lowtemperature transient PL microscopy. Focusing on the linear regime of low-excitation densities, we find a fast and unconventional, time-dependent exciton diffusion with an initial increase of the diffusion coefficient of up to $50 \mathrm{~cm}^{2} \mathrm{~s}^{-1}$ stemming from hot dark excitons with substantial excess energies. This rapid expansion is followed by thermalization and cooling of the exciton distribution on a timescale of a few tens of picoseconds, converging towards a steady-state diffusivity of about 2 to $3 \mathrm{~cm}^{2} \mathrm{~s}^{-1}$. Our findings provide fundamental insights into the non-equilibrium transport of excitons in atomically thin semiconductors with potential implications towards their technological application in optoelectronic devices.

\section{Conflicts of interest}

There are no conflicts to declare.

\section{Acknowledgements}

We thank Mikhail M. Glazov (Ioffe Institute) for valuable discussions on exciton diffusion. This project has received funding from the European Union's Horizon 2020 Research and Innovation programme under grant agreement no. 881603 (Graphene Flagship) and from the 2D Tech VINNOVA competence Center (Ref. 2019-00068). The computations were enabled by resources provided by the Swedish National Infrastructure for Computing (SNIC). Financial support by the DFG via SFB 1083 (project B9) and SFB 1244 (project B05), SPP 2196 Priority Program ( $\mathrm{CH}$ 1672/3-1) and Emmy Noether Initiative (CH 1672/1, Project-ID: 287022282), and the Würzburg-Dresden Cluster of Excellence on Complexity and Topology in Quantum Matter ct.qmat (EXC 2147, Project-ID: 390858490) is gratefully acknowledged. K. W. and T. T. acknowledge support from the Elemental Strategy Initiative conducted by the MEXT, Japan, grant number JPMXP0112101001, JSPS KAKENHI grant numbers JP20H00354 and the CREST (JPMJCR15F3), JST.

\section{Notes and references}

1 G. Wang, A. Chernikov, M. M. Glazov, T. F. Heinz, X. Marie, T. Amand and B. Urbaszek, Rev. Mod. Phys., 2018, 90, 021001. 2 T. Mueller and E. Malic, npj 2D Mater. Appl., 2018, 2, 29.
3 K. F. Mak, C. Lee, J. Hone, J. Shan and T. F. Heinz, Phys. Rev. Lett., 2010, 105, 136805.

4 D. Xiao, G.-B. Liu, W. Feng, X. Xu and W. Yao, Phys. Rev. Lett., 2012, 108, 196802.

5 W. Zhao, Z. Ghorannevis, L. Chu, M. Toh, C. Kloc, P.-H. Tan and G. Eda, ACS Nano, 2013, 7, 791-797.

6 A. Chernikov, T. C. Berkelbach, H. M. Hill, A. Rigosi, Y. Li, O. B. Aslan, D. R. Reichman, M. S. Hybertsen and T. F. Heinz, Phys. Rev. Lett., 2014, 113, 076802.

7 X.-X. Zhang, Y. You, S. Y. F. Zhao and T. F. Heinz, Phys. Rev. Lett., 2015, 115, 257403.

8 M. Selig, G. Berghäuser, A. Raja, P. Nagler, C. Schüller, T. F. Heinz, T. Korn, A. Chernikov, E. Malic and A. Knorr, Nat. Commun., 2016, 7, 13279.

9 T. Chervy, S. Azzini, E. Lorchat, S. Wang, Y. Gorodetski, J. A. Hutchison, S. Berciaud, T. W. Ebbesen and C. Genet, ACS Photonics, 2018, 5, 1281-1287.

10 M. M. Fogler, L. V. Butov and K. S. Novoselov, Nat. Commun., 2014, 5, 4555.

11 P. Rivera, J. R. Schaibley, A. M. Jones, J. S. Ross, S. Wu, G. Aivazian, P. Klement, K. Seyler, G. Clark, N. J. Ghimire, J. Yan, D. G. Mandrus, W. Yao and X. Xu, Nat. Commun., 2015, 6, 6242.

12 P. Merkl, F. Mooshammer, P. Steinleitner, A. Girnghuber, K.-Q. Lin, P. Nagler, J. Holler, C. Schüller, J. M. Lupton, T. Korn, S. Ovesen, S. Brem, E. Malic and R. Huber, Nat. Mater., 2019, 18, 691-696.

13 W.-T. Hsu, L.-S. Lu, D. Wang, J.-K. Huang, M.-Y. Li, T.-R. Chang, Y.-C. Chou, Z.-Y. Juang, H.-T. Jeng, L.-J. Li and W.-H. Chang, Nat. Commun., 2017, 8, 929.

14 A. Steinhoff, M. Florian, M. Rösner, G. Schönhoff, T. O. Wehling and F. Jahnke, Nat. Commun., 2017, 8, 1166.

15 O. B. Aslan, M. Deng and T. F. Heinz, Phys. Rev. B, 2018, 98, 115308.

16 T. Deilmann and K. S. Thygesen, 2D Mater., 2019, 6, 035003.

17 G.-H. Peng, P.-Y. Lo, W.-H. Li, Y.-C. Huang, Y.-H. Chen, C.-H. Lee, C.-K. Yang and S.-J. Cheng, Nano Lett., 2019, 19, 2299-2312.

18 J. Madéo, M. K. L. Man, C. Sahoo, M. Campbell, V. Pareek, E. L. Wong, A. Al-Mahboob, N. S. Chan, A. Karmakar, B. M. K. Mariserla, X. Li, T. F. Heinz, T. Cao and K. M. Dani, Science, 2020, 370, 1199-1204.

19 S. Dong, M. Puppin, T. Pincelli, S. Beaulieu, D. Christiansen, H. Hübener, C. W. Nicholson, R. P. Xian, M. Dendzik, Y. Deng, Y. W. Windsor, M. Selig, E. Malic, A. Rubio, A. Knorr, M. Wolf, L. Rettig and R. Ernstorfer, Nat. Sci., 2021, 1, e10010.

20 R. Wallauer, R. Perea-Causin, L. Münster, S. Zajusch, S. Brem, J. Güdde, K. Tanimura, K.-Q. Lin, R. Huber, E. Malic and U. Höfer, Nano Lett., 2021, 21, 5867-5873.

21 E. Courtade, M. Semina, M. Manca, M. M. Glazov, C. Robert, F. Cadiz, G. Wang, T. Taniguchi, K. Watanabe, M. Pierre, W. Escoffier, E. L. Ivchenko, P. Renucci, X. Marie, T. Amand and B. Urbaszek, Phys. Rev. B, 2017, 96, 085302 . 
22 J. Lindlau, C. Robert, V. Funk, J. Förste, M. Förg, L. Colombier, A. Neumann, E. Courtade, S. Shree, T. Taniguchi, et al., 2017, arXiv:1710.00988, arXiv preprint.

23 Z. Ye, L. Waldecker, E. Y. Ma, D. Rhodes, A. Antony, B. Kim, X.-X. Zhang, M. Deng, Y. Jiang, Z. Lu, D. Smirnov, K. Watanabe, T. Taniguchi, J. Hone and T. F. Heinz, Nat. Commun., 2018, 9, 3718.

24 M. Barbone, A. R.-P. Montblanch, D. M. Kara, C. PalaciosBerraquero, A. R. Cadore, D. De Fazio, B. Pingault, E. Mostaani, H. Li, B. Chen, K. Watanabe, T. Taniguchi, S. Tongay, G. Wang, A. C. Ferrari and M. Atatüre, Nat. Commun., 2018, 9, 3721.

25 Z. Li, T. Wang, C. Jin, Z. Lu, Z. Lian, Y. Meng, M. Blei, S. Gao, T. Taniguchi, K. Watanabe, T. Ren, S. Tongay, L. Yang, D. Smirnov, T. Cao and S.-F. Shi, Nat. Commun., 2019, 10, 2469.

26 E. Liu, J. van Baren, T. Taniguchi, K. Watanabe, Y.-C. Chang and C. H. Lui, Phys. Rev. Res., 2019, 1, 032007.

27 M. He, P. Rivera, D. Van Tuan, N. P. Wilson, M. Yang, T. Taniguchi, K. Watanabe, J. Yan, D. G. Mandrus, H. Yu, H. Dery, W. Yao and X. Xu, Nat. Commun., 2020, 11, 618.

28 S. Brem, A. Ekman, D. Christiansen, F. Katsch, M. Selig, C. Robert, X. Marie, B. Urbaszek, A. Knorr and E. Malic, Nano Lett., 2020, 20, 2849-2856.

29 R. Rosati, K. Wagner, S. Brem, R. Perea-Causín, E. Wietek, J. Zipfel, J. D. Ziegler, M. Selig, T. Taniguchi, K. Watanabe, A. Knorr, A. Chernikov and E. Malic, ACS Photonics, 2020, 7, 2756-2764.

30 D. F. Cordovilla Leon, Z. Li, S. W. Jang and P. B. Deotare, Phys. Rev. B, 2019, 100, 241401.

31 M. Kulig, J. Zipfel, P. Nagler, S. Blanter, C. Schüller, T. Korn, N. Paradiso, M. M. Glazov and A. Chernikov, Phys. Rev. Lett., 2018, 120, 207401.

32 R. Perea-Causín, S. Brem, R. Rosati, R. Jago, M. Kulig, J. D. Ziegler, J. Zipfel, A. Chernikov and E. Malic, Nano Lett., 2019, 19, 7317-7323.

33 M. M. Glazov, Phys. Rev. B, 2019, 100, 045426.

34 T. Kato and T. Kaneko, ACS Nano, 2016, 10, 9687-9694.

35 F. Cadiz, C. Robert, E. Courtade, M. Manca, L. Martinelli, T. Taniguchi, K. Watanabe, T. Amand, A. C. H. Rowe, D. Paget, B. Urbaszek and X. Marie, Appl. Phys. Lett., 2018, 112, 152106.

36 S. Z. Uddin, H. Kim, M. Lorenzon, M. Yeh, D.-H. Lien, E. S. Barnard, H. Htoon, A. Weber-Bargioni and A. Javey, ACS Nano, 2020, 14, 13433-13440.

37 D. F. Cordovilla Leon, Z. Li, S. W. Jang, C.-H. Cheng and P. B. Deotare, Appl. Phys. Lett., 2018, 113, 252101.

38 J. Wang, Y. Guo, Y. Huang, H. Luo, X. Zhou, C. Gu and B. Liu, Appl. Phys. Lett., 2019, 115, 131902.

39 R. Rosati, R. Perea-Causín, S. Brem and E. Malic, Nanoscale, 2020, 12, 356-363.

40 M. G. Harats, J. N. Kirchhof, M. Qiao, K. Greben and K. I. Bolotin, Nat. Photonics, 2020, 14, 324-329.

41 H. Moon, G. Grosso, C. Chakraborty, C. Peng, T. Taniguchi, K. Watanabe and D. Englund, Nano Lett., 2020, 20, 67916797.
42 R. Rosati, S. Brem, R. Perea-Causín, R. Schmidt, I. Niehues, S. M. de Vasconcellos, R. Bratschitsch and E. Malic, 2D Mater., 2021, 8, 015030.

43 H. Zhao, B. Dal Don, S. Moehl, H. Kalt, K. Ohkawa and D. Hommel, Phys. Rev. B: Condens. Matter Mater. Phys., 2003, 67, 035306.

44 H. Kalt, H. Zhao, B. D. Don, G. Schwartz, C. Bradford and K. Prior, J. Lumin., 2005, 112, 136-141.

45 J. Sung, C. Schnedermann, L. Ni, A. Sadhanala, R. Y. S. Chen, C. Cho, L. Priest, J. M. Lim, H.-K. Kim, B. Monserrat, P. Kukura and A. Rao, Nat. Phys., 2020, 16, 171-176.

46 T. Hotta, S. Higuchi, A. Ueda, K. Shinokita, Y. Miyauchi, K. Matsuda, K. Ueno, T. Taniguchi, K. Watanabe and R. Kitaura, Phys. Rev. B, 2020, 102, 115424.

47 H. Haug and S. W. Koch, Quantum Theory of the Optical and Electronic Properties of Semiconductors, World Scientific Publishing Company, 5th edn, 2009.

48 T. C. Berkelbach, M. S. Hybertsen and D. R. Reichman, Phys. Rev. B: Condens. Matter Mater. Phys., 2015, 92, 085413.

49 A. Kormányos, G. Burkard, M. Gmitra, J. Fabian, V. Zólyomi, N. D. Drummond and V. Fal'ko, 2D Mater., 2015, 2, 022001.

50 C. Robert, T. Amand, F. Cadiz, D. Lagarde, E. Courtade, M. Manca, T. Taniguchi, K. Watanabe, B. Urbaszek and X. Marie, Phys. Rev. B, 2017, 96, 155423.

51 G. Wang, C. Robert, M. M. Glazov, F. Cadiz, E. Courtade, T. Amand, D. Lagarde, T. Taniguchi, K. Watanabe, B. Urbaszek and X. Marie, Phys. Rev. Lett., 2017, 119, 047401. 52 K. Wagner, J. Zipfel, R. Rosati, E. Wietek, J. D. Ziegler, S. Brem, R. Perea-Causín, T. Taniguchi, K. Watanabe, M. M. Glazov, E. Malic and A. Chernikov, Phys. Rev. Lett., 2021, 127, 076801.

53 O. Hess and T. Kuhn, Phys. Rev. A, 1996, 54, 3347-3359.

54 M. Kira and S. Koch, Prog. Quantum Electron., 2006, 30, 155-296.

55 F. Lengers, T. Kuhn and D. E. Reiter, Phys. Rev. B, 2020, 101, 155304.

56 Z. Jin, X. Li, J. T. Mullen and K. W. Kim, Phys. Rev. B: Condens. Matter Mater. Phys., 2014, 90, 045422.

57 I. Paradisanos, G. Wang, E. M. Alexeev, A. R. Cadore, X. Marie, A. C. Ferrari, M. M. Glazov and B. Urbaszek, Nat. Commun., 2021, 12, 538.

58 S. Brem, M. Selig, G. Berghaeuser and E. Malic, Sci. Rep., 2018, 8, 8238.

59 F. Steininger, A. Knorr, P. Thomas and S. W. Koch, . Phys. B: Condens. Matter, 1997, 103, 45-52.

60 A. Castellanos-Gomez, M. Buscema, R. Molenaar, V. Singh, L. Janssen, H. S. J. van der Zant and G. A. Steele, 2D Mater., 2014, 1, 011002.

61 G. Berghäuser, P. Steinleitner, P. Merkl, R. Huber, A. Knorr and E. Malic, Phys. Rev. B, 2018, 98, 020301(R).

62 G. Berghäuser, I. Bernal-Villamil, R. Schmidt, R. Schneider, I. Niehues, P. Erhart, S. Michaelis de Vasconcellos, R. Bratschitsch, A. Knorr and E. Malic, Nat. Commun., 2018, 9, 971. 